\title{
Prognostic Value of LDHD and Its Correlation with Immune Infiltrates in Thyroid Cancer
}

Jing Zhong ( $\square$ zhongjing2002@usc.edu.cn )

University of South China

Weiqiang Tang

University of South China

\section{Zuyao Chen}

University of South China

Yajie Liao

University of South China

Yuan Fang

Kunming Medical University

Min Xia

University of South China

Xuyu Zu

University of South China

Gebo Wen

University of South China

\section{Research Article}

Keywords: LDHD, lactate dehydrogenase D, prognosis, thyroid cancer, immune cell infiltration

Posted Date: April 5th, 2022

DOI: https://doi.org/10.21203/rs.3.rs-1395483/v2

License: (a) (i) This work is licensed under a Creative Commons Attribution 4.0 International License.

Read Full License 


\section{Abstract}

Background: LDHD is a subtype of the LDH family that can participate in D-lactate metabolism. However, the expression of LDHD in tumors and its role in tumor immunity are currently unknown. The objective of this research was to estimate the expression and prognosis of LDHD in pan-cancer and to explore the communication between LDHD expression level and tumor immune infiltration.

Methods: LDHD expression level in pan-cancer was explored by TIMER and UALCAN databases and the prognostic value of LDHD was explored by Kaplan Meier plotter and PrognoScan databases. Then, the correlation between LDHD expression level and immune cell infiltration was evaluated by the TIMER database. In addition, the relationship between LDHD expression and clinical features of THCA was verified in the TCGA database.

Results: The expression of LDHD mRNA or protein is downregulated in varied tumor types, and LDHD expression level is significantly related to the prognosis in tumors. Moreover, the downregulation of LDHD was found related to increased immune-cell infiltration and worse clinical features, especially in thyroid cancer.

Conclusion: LDHD may be a potential pan-cancer prognostic biomarker. In thyroid cancer, loss of expression of LDHD may mediate tumor initiation and progression by affecting immune cell infiltration.

\section{Background}

Thyroid cancer (THCA) is the commonest malignant tumor in the endocrine system, and the new cases of THCA steadily increased worldwide for the past thirty years. According to the estimation, 44280 new cases and 2200 deaths from THCA will occur in the United States in 2021 [1, 2]. The main treatment methods of THCA patients include surgery and radioiodine therapy, with an excellent overall prognosis [3]. Although most THCA patients can be cured after treatment, some still die of distant metastasis and advanced tumors. The existing therapies remain poorly curative for this subset of THCA patients with locally advanced or distant metastases. At present, the pathogenesis of THCA is not well understood, and ionizing radiation is the only well-established risk factor of THCA [4]. Accumulating evidence suggests that the recurrence and metastasis of THCA are partly attributed to the interaction of the tumor with the surrounding immune microenvironment that promotes immune tolerance, which in turn progresses to immune escape, resulting in the inability of the body to completely clear the tumor $[5,6]$.

The lactate dehydrogenase (LDH) family comprises four members: LDHA, LDHB, LDHC, and LDHD. Among them, LDHA, LDHB, and LDHC are responsible for the metabolism of L-lactate, while LDHD is responsible for the metabolism of D-lactate, an optical isomer of L-lactate [7]. Enzymatic loss of function in LDHD can lead to D-lactate accumulation in the body and cause D-lactic acidosis, hyperuricemia, and gout $[8,9]$. Previous studies indicated that LDHD can mediate the metabolism of D-Lactate and output newly synthesized malic acid in prostate cancer PC-3 cells [10]. In addition, it has been reported that LDHD expression is downregulated in clear cell renal cell carcinoma compared with normal tissues, and 
the downregulated LDHD expression is associated with a shorter OS and higher tumor grade [11]. These researches suggest that LDHD may play a vital role in cancer progression. However, there are few studies on the potential biological impact of LDHD in tumors, and the function of LDHD in tumorigenesis and its relevance with tumor immune cell infiltration remains unclear.

In this research, we thoroughly analyzed the expression of LDHD and the interrelationship of LDHD expression level and prognosis in multiple tumors via several databases, such as TIMER, PrognoScan, and GEPIA2. Furthermore, we explored the association of LDHD expression and tumor-infiltrating immune cells by the TIMER database. The findings of the present study elucidate that LDHD is downregulated in various tumors, which is significantly related to poorer prognosis in cancers. Not only that, we found LDHD expression was associated with different clinical features and immune infiltration in THCA. The loss of LDHD in THCA may contribute to the generation of immune infiltrates and tumorigenesis and progression, implying that LDHD is probably a potential biomarker for detection and immunotherapeutic target in THCA.

\section{Materials And Methods}

\section{Clinical Samples}

All THCA and para-cancerous tissues were surgically resected at First Affiliated Hospital of South China University with written informed consent. There are thirty patients with papillary thyroid carcinoma (PTC) and one patient with anaplastic thyroid carcinoma (ATC) undergoing surgical resection were enrolled. The included patients according as follows: 1 . over eighteen years old; 2 . All THCA patients were diagnosed by two senior pathologists and without other tumors. 3. sample was collected before patients received any chemoradiotherapy or immunotherapy.

\section{Real-Time PCR}

Using a Trizol based method to extract the total RNA from fresh PTC or para-carcinoma tissues. Primers for LDHD (forward, 5'-GTAGCTGACTGGTCACCCTG-3', reverse, 5'- CTCCCTTTGCCTTCTGGGAG-3') and GAPDH (forward, 5'-CAGGAGGCATTGCTGATGAT-3', reverse, 5'-GAAGGCTGGGGCTCATTT-3') were used for qRT-PCR.

\section{Western Blot}

THCA and para-cancerous tissue lysates were lysed on ice for $30 \mathrm{~min}$. Soluble protein $(30 \mu \mathrm{g}) \mathrm{was}$ separated on 10\% SDS/PAGE gels, and western blot was conducted using LDHD antibodies (14398-1-AP, ProteinTech Group, Chicago, IL, USA). The same membrane was reprobed for GAPDH, which served as the loading control for the experiment. 


\section{Immunohistochemistry (IHC) Staining}

Formalin-fixed THCA and para-cancerous tissues embedded in paraffin were used to determine LDHD expression. Images analysis of immunostained THCA and para-cancerous tissue slides were evaluated by two separate expert pathologists.

\section{UALCAN Database Analysis}

UALCAN database was used to investigate the LDHD protein expression levels in diverse tumors and compared normal tissues. Protein expression data were based on the CPTAC database, and P-value < 0.05 was considered statistically significant.

\section{PrognoScan Database Analysis}

PrognoScan database was applied to explore the prognostic value of LDHD for cancer patients (data source from GEO). Overall survival (OS) and Relapse Free Survival (RFS) were estimated via entering gene symbol "LDHD".

\section{Kaplan-Meier Plotter Database Analysis}

Kaplan-Meier Plotter was applied to investigate the prognostic value of LDHD in patients with several tumor types [12]. OS, RFS, FP (first progression), and PFS (progression-free survival) were assessed in those cancers.

\section{GEPIA2 Database Analysis}

GEPIA2 was based on TCGA and GTEx data and applied to investigate the prognostic value of LDHD. OS and RFS were evaluated via entering gene symbol "LDHD" and group cutoff set to "Median".

\section{TIMER Database Analysis}

Tumor Immune Estimation Resource (TIMER) database was applied to investigate the mRNA expression of LDHD in pan-cancer and the relationship of its expression level and immune infiltration[13, 14].

\section{ENCORI Database Analysis}

ENCORI database was applied to investigate the association in the expression of LDHD and immune checkpoint genes in THCA [15]. The expression data of cancers are from the TCGA project and $P$-value < 


\section{TILs profile}

Single sample gene set enrichment (sSGSEA) analysis using the GSVA package was used to assess the tumor-infiltrating lymphocytes (TILs) abundance profiles in TCGA-THCA samples. A total of 24 immune cell types were included in the correlation analysis, and enrichment scores were used to normalize [16].

\section{LinkedOmics Database Analysis}

LinkedOmics database involves genomic, transcriptomic, and clinical data for 32 TCGA tumor types [17]. LinkFinder module was applied to analyze the differentially expressed genes associated with LDHD in the TCGA-THCA cohort. LinkInterpreter module was applied to investigate the Gene Ontology biological process (GO_BP) and Kyoto Encyclopedia of Genes and Genomes (KEGG) pathways by the gene set enrichment analysis (GSEA).

\section{Results}

\section{LDHD mRNA expression levels across different cancers}

To clarify contrasts of the expression of LDHD in tumor and normal tissues, the mRNA expression of LDHD in human cancers was investigated via the TIMER online database. The results showed that the mRNA expression of LDHD was stronger in kidney chromophobe $(\mathrm{KICH})$ compared with normal tissue. Moreover, LDHD expression was significantly lower in fifteen cancer types, including THCA, compared to normal tissues (Figure 1A).

\section{LDHD protein expression levels across different cancers}

To further verify the protein expression levels of LDHD in cancerous and normal tissues, the LDHD protein expression levels in 10 cancer types and corresponding normal tissues were explored by UALCAN online database. The results showed that the protein levels of LDHD in breast invasive carcinoma, colon adenocarcinoma, glioblastoma multiforme, head and neck squamous cell carcinoma, liver hepatocellular carcinoma, lung adenocarcinoma, ovarian cancer, pancreatic adenocarcinoma, and kidney renal clear cell carcinoma were significantly lower than in corresponding normal tissues, while there was nonsignificance in uterine corpus endometrial carcinoma (Figure 1B-K). These findings illustrated that LDHD expression is significantly downregulated in various tumors and suggest that loss of LDHD may play an essential regulatory role in tumor progression.

\section{Prognostic value of LDHD in different cancers}




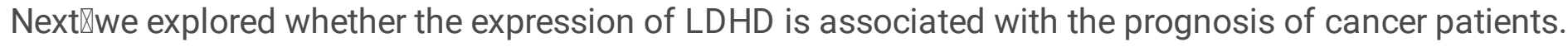
The effect of LDHD expression on survival was assessed using PrognoScan. The results displayed that the LDHD expression level was significantly associated with prognosis in four GEO data set: OS (HR = $0.65 ; P=0.013203)$ and RFS (HR $=0.70 ; P=0.008830)$ in lung adenocarcinoma cohort GSE31210 $(n=204)$, OS (HR $=0.71 ; P=0.015131)$ in lung adenocarcinoma cohort GSE13213 ( $n=117)$, OS $(H R=0.13$; $P=0.015291)$ in renal cell carcinoma cohort E-DKFZ-1 ( $n=59)$, OS (HR, $0.15 ; P=0.046356)$ in acute myoid leukemia cohort GSE12417-GPL570 ( $n=79$ ) (Figure 2 A-E).

The same work was applied in the Kaplan Meier plotter database to analyze the association of LDHD expression with breast cancer, gastric cancer, lung cancer and ovarian cancer, liver cancer prognosis, and $\mathrm{OS}$ and RFS were used as indicators to judge the prognostic value (Figure $2 \mathrm{~F}-0$ ). In all five tumors, low expression of LDHD was a poor prognostic factor: lung cancer (OS: $\mathrm{HR}=0.55 ; P=2 \times 10^{-12}$; FP: $\mathrm{HR}=0.56$; $P=1.6 \times 10^{-5}$ ), ovarian cancer (OS: HR $=0.75 ; P=0.017$; PFS: HR $\left.=0.78 ; P=0.013\right)$, breast cancer(OS: HR $=0.79 ; 95 \% \mathrm{Cl}, 0.6-1.06 ; P=0.12 ; \mathrm{RFS}: \mathrm{HR}=0.81 ; P=0.012)$, gastric cancer(OS: $\mathrm{HR}=0.62 ; P=1.5 \times 10^{-5}$; FP: HR, 0.78; $P=0.00022$ ), liver cancer (OS: HR $=0.55 ; P=0.0014$; RFS: HR= 0.68; $P=0.02$ ).

In addition, the prognostic potential of LDHD in more tumor types was also investigated by the GEPIA2 database. The cutoff value was set as "median" and the filter was set as a $P<0.05$ and $P(\mathrm{HR})<0.05$. The results displayed that LDHD expression was significant association with the prognosis in multiple cancer types (Figure S1). Interestingly, in common with our results above, the low LDHD expression level in various other tumors was significantly related to a poorer prognosis. Those results have impressive consistency and confirm LDHD is a protective factor in multiple types of cancer. In contrast, loss of LDHD in tumors may contribute to tumor progression and poor prognosis.

\section{LDHD influenced the extent of immune infiltration in THCA}

There is a close relationship between lactate metabolism and tumor-infiltrating lymphocytes in tumors. We speculate that LDHD, a lactate metabolism gene, may also affect tumor immune infiltration. To clarify the effect of LDHD expression in TIL, the Spearman correlation values of LDHD expression with various immune cell infiltration levels were obtained in the TIMER database. The results revealed that LDHD expression was significantly associated with immune cell infiltration in multiple tumors, while the absolute value of the correlation was highest in THCA (Figure 3A-C). The LDHD expression level was significantly negative association with B cells, CD4+ T cells, macrophage, neutrophil, and dendritic cells infiltration level in THCA. The association of LDHD expression level with the ImmuneScore, ESTIMATEScore, and StromalScore was calculated from expression profiling data using the ESTIMATE R package (Figure 3D-F).

To gain insight into the possible role of LDHD in various immune cell infiltrates in THCA, we used the TIMER database to explore the relationship between LDHD and several immune marker sets that are widely accepted as corresponding symbols for different immune cells, such as B cell, T cell (general), 
CD8+ T cell and others (Table 1). The results show that the levels of most immune subsets that label different immune cells negatively correlate with the expression of LDHD in THCA. The results similarly showed a significant inverse association of the expression of LDHD with the immune infiltration level. The association of LDHD with immune checkpoints was also examined in the ENCORI database and the expression of LDHD was significantly inversely related to the expression of the four major immune checkpoints: CTLA-4, PD-1 (PDCD1), PD-L1 (CD274), PD-L2(PDCDL2) in THCA (Figure S2).

Moreover, we use the GEVA R package to calculate the association of LDHD expression level with 24 immune cells type infiltration (Figure 4). A total of 8 immune cell infiltration levels were correlated with

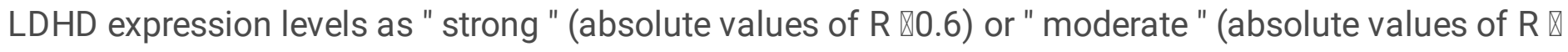
$0.4)$.

\section{Table 1}

\section{Analysis of the correlation between LDHD and gene markers of immune cells in THCA.}




\begin{tabular}{|c|c|c|c|c|c|}
\hline \multirow[t]{2}{*}{ Cell type } & \multirow[t]{2}{*}{ Marker } & \multicolumn{2}{|l|}{ None } & \multicolumn{2}{|l|}{ Purity } \\
\hline & & Cor & $P$ & Cor & $P$ \\
\hline \multirow[t]{3}{*}{ B cell } & CD19 & -0.240 & $\star \star \star *$ & -0.235 & $\star * *$ \\
\hline & $\mathrm{CD} 27$ & 0.147 & $\star *$ & 0.124 & * \\
\hline & CD79A & -0.314 & $\star * *$ & -0.305 & $\star * *$ \\
\hline \multirow[t]{3}{*}{ T cell (general) } & CD3D & -0.394 & $\star * *$ & -0.39 & $\star * *$ \\
\hline & CD3E & -0.358 & $\star * \star$ & -0.358 & $\star * *$ \\
\hline & $\mathrm{CD} 2$ & -0.362 & $\star \star \star *$ & -0.361 & $\star \star \star *$ \\
\hline \multirow[t]{2}{*}{ CD8+ T cell } & CD8A & -0.119 & * & -0.124 & * \\
\hline & CD8B & -0.343 & $\star \star \star$ & -0.352 & $\star \star \star$ \\
\hline \multirow[t]{3}{*}{ Tfh } & CXCR3 & -0.250 & $\star \star \star$ & -0.251 & $\star \star \star$ \\
\hline & CXCR5 & -0.299 & $\star \star \star$ & -0.286 & $\star \star \star$ \\
\hline & ICOS & -0.403 & $\star \star \star *$ & -0.398 & $\star \star \star$ \\
\hline \multirow[t]{5}{*}{ Th1 } & IFN-Y & -0.200 & $\star \star \star$ & -0.205 & $* \star *$ \\
\hline & STAT4 & -0.52 & $\star \star \star \star$ & -0.521 & $* \star *$ \\
\hline & STAT1 & -0.531 & $\star \star \star$ & -0.525 & $\star * \star$ \\
\hline & CD94 & -0.158 & $\star \star$ & -0.162 & $\star \star$ \\
\hline & BET & -0.14 & * & -0.146 & * \\
\hline \multirow[t]{5}{*}{ Th2 } & STAT6 & -0.246 & 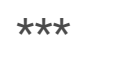 & -0.232 & $\star \star \star *$ \\
\hline & CD4 & -0.353 & 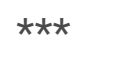 & -0.345 & $\star \star \star *$ \\
\hline & GATA-3 & -0.387 & $\star \star \star$ & -0.386 & $\star \star \star$ \\
\hline & CD184 & -0.212 & $\star \star \star$ & -0.216 & $\star \star \star$ \\
\hline & CD194 & -0.409 & $\star \star \star$ & -0.398 & $\star \star \star *$ \\
\hline \multirow[t]{3}{*}{ Th9 } & TGFBR2 & 0.223 & $\star \star \star *$ & 0.231 & $\star \star \star$ \\
\hline & IRF4 & -0.056 & 0.211 & -0.048 & 0.292 \\
\hline & SPI1 & -0.472 & $\star \star \star \star$ & -0.463 & $\star * *$ \\
\hline \multirow[t]{3}{*}{ Th17 } & CD161 & -0.345 & $\star \star \star \star$ & -0.345 & $\star \star \star *$ \\
\hline & CD121A & -0.449 & $\star \star *$ & -0.439 & $\star \star \star *$ \\
\hline & STAT3 & -0.308 & $\star \star *$ & -0.293 & $\star * *$ \\
\hline
\end{tabular}




\begin{tabular}{|c|c|c|c|c|c|}
\hline \multirow[t]{2}{*}{ Th22 } & CCR10 & -0.031 & 0.491 & -0.04 & 0.382 \\
\hline & AHR & -0.627 & 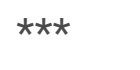 & -0.619 & $\star \star \star *$ \\
\hline \multirow[t]{4}{*}{ Treg } & CD25 & -0.518 & 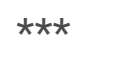 & -0.505 & $\star \star \star *$ \\
\hline & CCR8 & -0.387 & $\star \star \star ~$ & -0.378 & $\star \star \star$ \\
\hline & FOXP3 & -0.478 & $\star \star \star$ & -0.472 & $\star \star \star$ \\
\hline & CD127 & -0.371 & $\star \star \star$ & -0.358 & $\star \star \star$ \\
\hline \multirow[t]{5}{*}{ Exhausted $\mathrm{T}$ cell } & PD-1 & -0.113 & * & -0.135 & * \\
\hline & Tim-3 & -0.428 & $\star \star \star$ & -0.417 & $\star * \star$ \\
\hline & CTLA4 & -0.452 & $\star \star \star$ & -0.448 & $\star \star \star$ \\
\hline & LAG3 & -0.277 & $\star \star \star *$ & -0.281 & $\star \star \star *$ \\
\hline & GZMB & -0.215 & $\star \star \star$ & -0.222 & $\star * \star$ \\
\hline \multirow[t]{3}{*}{ M1 Macrophage } & INOS & 0.019 & 0.668 & 0.018 & 0.686 \\
\hline & IRF5 & -0.527 & $\star \star \star \star$ & -0.527 & 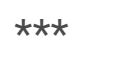 \\
\hline & $\operatorname{cox} 2$ & -0.582 & 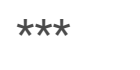 & -0.579 & $\star \star \star ~$ \\
\hline \multirow[t]{3}{*}{ M2 Macrophage } & CD163 & -0.366 & 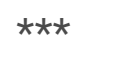 & -0.35 & $\star \star \star *$ \\
\hline & VSIG4 & -0.347 & 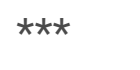 & -0.335 & $\star \star \star *$ \\
\hline & MS4A4A & -0.388 & $\star \star \star *$ & -0.372 & $\star \star \star \star$ \\
\hline \multirow[t]{3}{*}{ TAM } & CD80 & -0.414 & 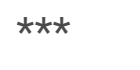 & -0.407 & $\star \star \star *$ \\
\hline & CCL2 & -0.336 & $\star \star \star *$ & -0.331 & $\star \star \star *$ \\
\hline & IL10 & -0.322 & $\star \star \star *$ & -0.305 & $* * *$ \\
\hline \multirow[t]{2}{*}{ Monocyte } & CD86 & -0.456 & $\star \star \star \star$ & -0.446 & $* \star \star$ \\
\hline & CD115 & -0.365 & $\star \star \star *$ & -0.357 & $\star \star * *$ \\
\hline \multirow[t]{7}{*}{ NK cell } & NCAM1 & 0.432 & $\star \star \star \star$ & 0.418 & $\star \star \star *$ \\
\hline & KIR2DL1 & 0.113 & 0.011 & 0.093 & 0.0395 \\
\hline & KIR2DL3 & -0.027 & 0.543 & -0.036 & 0.429 \\
\hline & KIR2DL4 & 0.082 & 0.063 & 0.081 & 0.0752 \\
\hline & KIR3DL1 & 0.068 & 0.124 & 0.057 & 0.205 \\
\hline & KIR3DL2 & -0.085 & 0.056 & -0.084 & 0.064 \\
\hline & KIR3DL3 & -0.025 & 0.575 & -0.035 & 0.435 \\
\hline
\end{tabular}




\begin{tabular}{|c|c|c|c|c|c|}
\hline & KIR2DS4 & -0.003 & 0.947 & -0.009 & 0.848 \\
\hline & CD94 & -0.158 & ** & -0.162 & ** \\
\hline & CD314 & -0.194 & 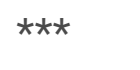 & -0.202 & $\star \star \star *$ \\
\hline \multirow[t]{5}{*}{ Neutrophil } & CD66b & -0.335 & 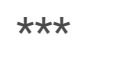 & -0.33 & $\star \star \star *$ \\
\hline & CD11b & -0.428 & $\star \star \star *$ & -0.416 & $\star \star \star$ \\
\hline & CD15 & -0.127 & * & -0.107 & 0.018 \\
\hline & CCR7 & -0.372 & $\star \star \star$ & -0.361 & $\star \star \star *$ \\
\hline & MPO & -0.013 & 0.772 & -0.029 & 0.517 \\
\hline \multirow[t]{8}{*}{ Dendritic cell } & CD1C & -0.534 & $\star \star \star$ & -0.527 & $\star \star \star$ \\
\hline & CD141 & 0.131 & * & 0.128 & * \\
\hline & HLA-DPB1 & -0.394 & $\star \star \star$ & -0.387 & $\star * \star$ \\
\hline & HLA-DQB1 & -0.4 & $\star \star \star$ & -0.398 & $\star \star \star$ \\
\hline & HLA-DRA & -0.424 & $\star \star \star \star$ & -0.413 & 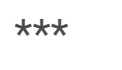 \\
\hline & HLA-DPA1 & -0.414 & $\star \star \star \star$ & -0.408 & $\star \star \star ~$ \\
\hline & BDCA-4 & -0.007 & 0.881 & 0 & 0.994 \\
\hline & CD11c & -0.402 & 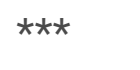 & -0.394 & $\star \star \star *$ \\
\hline $\begin{array}{l}* \mathrm{P}<0.05 \\
* * \mathrm{P}<0.001 \\
* * * \mathrm{P}<0.0001\end{array}$ & & & & & \\
\hline
\end{tabular}

\section{Enrichment analysis of LDHD in THCA}

The LinkFinder module in the LinkedOmics web portal was used to analyze the co-expression pattern of LDHD in TCGA-THCA (Figure 5A-C). GO term annotation indicated genes negatively related to LDHD join mainly in adaptive immune response, leukocyte cell-cell adhesion, lymphocyte activation involved in immune response, regulation of innate immune response, $T$ cell activation (Figure 5D).

KEGG pathway analysis displayed that the genes negatively associated with LDHD join mainly in Inflammatory bowel disease (IBD), Cell adhesion molecules (CAMs), Cytokine-cytokine receptor interaction, Th17 cell differentiation, Complement and coagulation cascades (Figure 5E). The above results further confirmed that downregulation of LDHD in THCA involved immune infiltration in promoting tumor progression of THCA. 


\section{LDHD Expression and relationship with clinical features in THCA}

Considering the close correlation between immune infiltration and tumor progression, we explored the correlation of LDHD expression with clinical features in THCA. We grouped THCA according to their different clinical characteristics and analyzed the expression of LDHD among different groups. The results showed the absence of LDHD was significantly related to higher T stage, lymph node metastasis, higher TNM stage, extrathyroidal extension, and no significant correlation with clinical characteristics such as age, distant metastasis, or gender (Figure 6). That result suggests that the loss of LDHD may be critical for the development of THCA.

\section{Decreased Expression of LDHD in THCA Patients}

We examined LDHD expression in the collected tissues of 30 PTC and adjacent noncancerous tissues via qRT-PCR. As in the TCGA database, our qRT-PCR results similarly showed that the LDHD mRNA expression was significantly weaker in tumors than in adjacent noncancerous tissues (Figure 7A). Meanwhile, LDHD was absent to a greater extent in PTC patients with lymph node metastasis (Figure 7B). The above results revealed that loss of LDHD might play a critical role in both tumorigenesis and metastasis. Next, the protein expression of LDHD in THCA and matched tissues was assessed via WB and IHC (Figure 7C-D). The western blot also revealed that the protein expression of LDHD was also significantly stronger in cancer than matched normal tissues.

\section{Discussion}

Although tumor therapy methods have greatly advanced over the past few decades, cancer remains the most severe threat to human life and health globally [18]. Early detection and effective treatment are relevant to improving the prognosis of cancer patients. Hence, identifying sensitive biomarkers is crucial for the early diagnosis and prognosis determination of cancer. This study provides the first exhaustively analyzed the expression of LDHD in pan-cancer and found that LDHD appears aberrantly expressed in various tumors. The mRNA expression analysis from the TCGA dataset and protein expression analysis from the CPTAC dataset showed that LDHD is significantly downregulated in most tumor types. Survival analysis revealed that low expression of LDHD in multiple tumor types was related to worse OS, RFS, PFS, and FP, suggesting that LDHD may be a tumor suppressor in Pan-cancer.

Cancer cells are highly dependent on glycolysis and aerobic glycolysis results in the conversion of as much as $85 \%$ glucose converted to lactate and released into the extracellular, which is called the Warburg effect [19-21]. The LDH enzyme plays a major role in glucose metabolism and is involved in the reduction and oxidation reactions between pyruvate and lactate [22]. Previous views considered that lactate is a waste product of glycolysis, but with advances in metabolomics, increasing studies evidence that lactate played a vital role in cell metabolism and immunity [23]. Lactate secreted by tumor and 
stromal cells can promote tumor progression by acidifying the tumor microenvironment (TME). The extracellular $\mathrm{pH}$ of solid tumors is acidic, whereas that of normal tissues is significantly more alkaline. Tumor extracellular acidosis resulting from lactate production significantly suppresses $T$ cell-mediated tumor immune responses [24-27]. On the other hand, lactate secretion induces VEGF expression, thus leading to angiogenesis and ensuring adequate oxygen and nutrient supply for proliferation in tumors $[28,29]$. In addition, lactate can act as a raw material for the tricarboxylic acid (TCA) cycle in cancer cells and is also a requirement precursor for tumor biosynthesis [30, 31]. LDHD is essential for human Dlactate metabolism, loss of LDHD elevates D-lactate concentrations and causes D-lactate acidosis. We speculate that the loss of LDHD in tumors may cause the accumulation of D-lactate in the tumor microenvironment, which affects immune cells infiltration and lead to tumor progression. Given the close relationship between lactate metabolism and immune infiltration, we analyzed the association between LDHD expression levels and immune cell infiltration. According to the analysis result, LDHD expression levels were significantly relevant with immune cell infiltration in various tumor types, and the most relevant type was THCA. LDHD expression levels were negatively related to immune cells infiltration in THCA, including B cells, CD4 + T cells, macrophage, neutrophil, and dendritic cells. The analysis of the LinkedOmics database further pointed out that genes negatively associated with LDHD in THCA were mainly enriched in immune-related pathways. ESTIMATEScore and SSGSEA analyses similarly suggested a significant inverse correlation between LDHD expression and immune infiltration. All signs indicate that loss of LDHD is related to the immune infiltration process of THCA.

The role of TILs in the outcome of THCA has been controversial, with a more global view that immune cells activated in the TME secrete proinflammatory cytokines and chemokines that promote tumor cell proliferation [32]. Immune checkpoints are a class of immunosuppressive molecules that correlate with tumor development and progression. Due to the progress of immune checkpoint blockade (ICB) therapies, tumor immunotherapy has received much attention in recent decades [33]. Preclinical research has shown the potential role of small molecule inhibitors blocking the PD-1/PD-L1 axis in advanced THCA [34-36]. But most patients have few benefits after treatment with PD-1/PD-L1 inhibitors, for whom more effective therapeutic targets and predictive biomarkers of efficacy are still needed. Our study suggests that LDHD expression was significantly inversely related to the expression of several immune checkpoints in THCA, for instance, CTLA4, PD-1, and PD-L1. Loss of L Loss of LDHD was also positively associated with higher tumor stage and lymph node metastasis in THCA, suggesting that LDHD may promote the evolution and transformation of THCA by affecting the immune infiltration profile.

Collectively, our findings demonstrate that LDHD may be a tumor suppressor gene in various tumor types and highlight the value that detection of the expression of LDHD has for prognostic evaluation in THCA, and restoring the expression of LDHD might be a new direction of THCA treatment. We mainly researched the interrelationship of LDHD expression level and immune infiltration in THCA and reasonably demonstrated that LDHD negatively regulated immune infiltration in THCA. We also found that downregulation of LDHD in THCA leads to a worsening of clinical features (primary tumor scope, lymph node metastasis, pathological stage of tumor). We confirmed the low expression of LDHD in THCA by PCR, WB, and IHC assays, and detection of its expression level allowed assessment of tumor malignancy. 
But more studies at cellular and molecular levels are still needed to elucidate how LDHD affects the development and progression of tumors. Future studies focusing on the mechanism of LDHD in tumor development may provide novel targeted therapeutic strategies.

\section{Declarations}

\section{Ethics approval and consent to participate}

The study was conducted in accordance with the Declaration of Helsinki, and the protocol was approved by the Ethics Committee of the First Affiliated Hospital of South China University. All of the enrolled patients signed informed consent forms.

\section{Consent for publication}

Not applicable.

\section{Availability of data and materials}

The data underlying this study are freely available from TCGA data portal (https://portal.gdc.cancer.gov/projects/TCGA-THCA) and GEO dataset (http://www.ncbi.nlm.nih.gov/geo/) . The authors did not have special access privileges.

\section{Competing interests}

The authors declare that they have no competing interests.

\section{Funding}

This work was supported by the grants from the Natural Science Foundation of Hunan Province (No. 2019JJ20014), the National Natural Science Foundation of China (No. 81773294), the Program of National Health and Family Planning Commission of Hunan (20201929), the Chuanshan Talent Project of the University of South China (No. CS2018-3-35), the Natural Science Foundation of Hunan Province (No.2019JJ50541), and the Program of Hunan Health Commission (202207014277).

\section{Authors' contributions}

WT analyzed the data and drafted the manuscript. ZC and XZ contribute to the experimental part of this paper. YF, YL, and MX helped to design the relevant pictures and tables. JZ and GW reviewed the manuscript and revised it. All authors contributed to the manuscript and approved its submission.

\section{Acknowledgements}

Not applicable.

\section{Author details}


${ }^{1}$ Institute of Clinical Medicine, The First Affiliated Hospital, Hengyang Medical School, University of South China, Hengyang, Hunan, 421001, PR China

2 Institute of Pharmacy and Pharmacology, The First People's Hospital of Chenzhou, University of South China, Hengyang, Hunan, 421001, PR China

${ }^{3}$ Organ Transplantation Center, The First Affiliated Hospital, Kunming Medical University, Kunming, Yunnan, 650032, PR China

${ }^{4}$ Cancer Research Institute, The First Affiliated Hospital, Hengyang Medical School, University of South China, Hengyang, Hunan 421001, PR China

${ }^{5}$ Department of Metabolism and Endocrinology, The First Affiliated Hospital, Hengyang Medical School, University of South China, Hengyang, Hunan 421001, PR China

\section{References}

1. C. La Vecchia, M. Malvezzi, C. Bosetti, W. Garavello, P. Bertuccio, F. Levi, E. Negri, Thyroid cancer mortality and incidence: a global overview, International journal of cancer, 136 (2015) 2187-2195.

2. J. Kim, J.E. Gosnell, S.A. Roman, Geographic influences in the global rise of thyroid cancer, Nature reviews. Endocrinology, 16 (2020) 17-29.

3. S. Filetti, C. Durante, D. Hartl, S. Leboulleux, L.D. Locati, K. Newbold, M.G. Papotti, A. Berruti, Thyroid cancer: ESMO Clinical Practice Guidelines for diagnosis, treatment, and follow-upt, Annals of oncology : official journal of the European Society for Medical Oncology, 30 (2019) 1856-1883.

4. H. Sung, J. Ferlay, R.L. Siegel, M. Laversanne, I. Soerjomataram, A. Jemal, F. Bray, Global Cancer Statistics 2020: GLOBOCAN Estimates of Incidence and Mortality Worldwide for 36 Cancers in 185 Countries, CA: a cancer journal for clinicians, 71 (2021) 209-249.

5. Z. Xie, X. Li, Y. He, S. Wu, S. Wang, J. Sun, Y. He, Y. Lun, J. Zhang, Immune Cell Confrontation in the Papillary Thyroid Carcinoma Microenvironment, Frontiers in endocrinology, 11 (2020) 570604.

6. S.M. Ferrari, P. Fallahi, M.R. Galdiero, I. Ruffilli, G. Elia, F. Ragusa, S.R. Paparo, A. Patrizio, V. Mazzi, G. Varricchi, G. Marone, A. Antonelli, Immune and Inflammatory Cells in Thyroid Cancer Microenvironment, International journal of molecular sciences, 20 (2019).

7. L. de Bari, A. Atlante, T. Armeni, M.P. Kalapos, Synthesis and metabolism of methylglyoxal, S-Dlactoylglutathione and D-lactate in cancer and Alzheimer's disease. Exploring the crossroad of eternal youth and premature aging, Ageing research reviews, 53 (2019) 100915.

8. G.R. Monroe, A.M. van Eerde, F. Tessadori, K.J. Duran, S.M.C. Savelberg, J.C. van Alfen, P.A. Terhal, S.N. van der Crabben, K.D. Lichtenbelt, S.A. Fuchs, J. Gerrits, M.J. van Roosmalen, K.L. van Gassen, M. van Aalderen, B.G. Koot, M. Oostendorp, M. Duran, G. Visser, T.J. de Koning, F. Calì, P. Bosco, K. Geleijns, M.G.M. de Sain-van der Velden, N.V. Knoers, J. Bakkers, N.M. Verhoeven-Duif, G. van 
Haaften, J.J. Jans, Identification of human D lactate dehydrogenase deficiency, Nature communications, 10 (2019) 1477.

9. M. Drabkin, Y. Yogev, L. Zeller, R. Zarivach, R. Zalk, D. Halperin, O. Wormser, E. Gurevich, D. Landau, R. Kadir, Y. Perez, O.S. Birk, Hyperuricemia and gout caused by missense mutation in d-lactate dehydrogenase, The Journal of clinical investigation, 129 (2019) 5163-5168.

10. L. de Bari, L. Moro, S. Passarella, Prostate cancer cells metabolize d-lactate inside mitochondria via a D-lactate dehydrogenase which is more active and highly expressed than in normal cells, FEBS letters, 587 (2013) 467-473.

11. Y. Wang, G. Li, F. Wan, B. Dai, D. Ye, Prognostic value of D-lactate dehydrogenase in patients with clear cell renal cell carcinoma, Oncology letters, 16 (2018) 866-874.

12. B. Győrffy, Survival analysis across the entire transcriptome identifies biomarkers with the highest prognostic power in breast cancer, Computational and structural biotechnology journal, 19 (2021) 4101-4109.

13. B. Li, E. Severson, J.C. Pignon, H. Zhao, T. Li, J. Novak, P. Jiang, H. Shen, J.C. Aster, S. Rodig, S. Signoretti, J.S. Liu, X.S. Liu, Comprehensive analyses of tumor immunity: implications for cancer immunotherapy, Genome biology, 17 (2016) 174.

14. T. Li, J. Fan, B. Wang, N. Traugh, Q. Chen, J.S. Liu, B. Li, X.S. Liu, TIMER: A Web Server for Comprehensive Analysis of Tumor-Infiltrating Immune Cells, Cancer research, 77 (2017) e108-e110.

15. J.H. Li, S. Liu, H. Zhou, L.H. Qu, J.H. Yang, starBase v2.0: decoding miRNA-ceRNA, miRNA-ncRNA and protein-RNA interaction networks from large-scale CLIP-Seq data, Nucleic acids research, 42 (2014) D92-97.

16. G. Bindea, B. Mlecnik, M. Tosolini, A. Kirilovsky, M. Waldner, A.C. Obenauf, H. Angell, T. Fredriksen, L. Lafontaine, A. Berger, P. Bruneval, W.H. Fridman, C. Becker, F. Pagès, M.R. Speicher, Z. Trajanoski, J. Galon, Spatiotemporal dynamics of intratumoral immune cells reveal the immune landscape in human cancer, Immunity, 39 (2013) 782-795.

17. S.V. Vasaikar, P. Straub, J. Wang, B. Zhang, LinkedOmics: analyzing multi-omics data within and across 32 cancer types, Nucleic acids research, 46 (2018) D956-d963.

18. P. Gotwals, S. Cameron, D. Cipolletta, V. Cremasco, A. Crystal, B. Hewes, B. Mueller, S. Quaratino, C. Sabatos-Peyton, L. Petruzzelli, J.A. Engelman, G. Dranoff, Prospects for combining targeted and conventional cancer therapy with immunotherapy, Nature reviews. Cancer, 17 (2017) 286-301.

19. G.A. Brooks, The Science and Translation of Lactate Shuttle Theory, Cell metabolism, 27 (2018) 757785.

20. W.H. Koppenol, P.L. Bounds, C.V. Dang, Otto Warburg's contributions to current concepts of cancer metabolism, Nature reviews. Cancer, 11 (2011) 325-337.

21. M.G. Vander Heiden, L.C. Cantley, C.B. Thompson, Understanding the Warburg effect: the metabolic requirements of cell proliferation, Science (New York, N.Y.), 324 (2009) 1029-1033.

22. S. Van Wilpe, R. Koornstra, M. Den Brok, J.W. De Groot, C. Blank, J. De Vries, W. Gerritsen, N. Mehra, Lactate dehydrogenase: a marker of diminished antitumor immunity, Oncoimmunology, 9 (2020) 
1731942.

23. J.R. Doherty, J.L. Cleveland, Targeting lactate metabolism for cancer therapeutics, The Journal of clinical investigation, 123 (2013) 3685-3692.

24. M. Certo, C.H. Tsai, V. Pucino, P.C. Ho, C. Mauro, Lactate modulation of immune responses in inflammatory versus tumour microenvironments, Nature reviews. Immunology, 21 (2021) 151-161.

25. A. Ibrahim-Hashim, V. Estrella, Acidosis and cancer: from mechanism to neutralization, Cancer metastasis reviews, 38 (2019) 149-155.

26. X. Zhang, Y. Lin, R.J. Gillies, Tumor pH and its measurement, Journal of nuclear medicine : official publication, Society of Nuclear Medicine, 51 (2010) 1167-1170.

27. V. Huber, C. Camisaschi, A. Berzi, S. Ferro, L. Lugini, T. Triulzi, A. Tuccitto, E. Tagliabue, C. Castelli, L. Rivoltini, Cancer acidity: An ultimate frontier of tumor immune escape and a novel target of immunomodulation, Seminars in cancer biology, 43 (2017) 74-89.

28. F. Hirschhaeuser, U.G. Sattler, W. Mueller-Klieser, Lactate: a metabolic key player in cancer, Cancer research, 71 (2011) 6921-6925.

29. C. Pinheiro, A. Longatto-Filho, R. Nogueira, F. Schmitt, F. Baltazar, Lactate-induced IL-8 pathway in endothelial cells--letter, Cancer research, 72 (2012) 1901-1902; author reply 1903-1904.

30. S. Hui, J.M. Ghergurovich, R.J. Morscher, C. Jang, X. Teng, W. Lu, L.A. Esparza, T. Reya, Z. Le, J. Yanxiang Guo, E. White, J.D. Rabinowitz, Glucose feeds the TCA cycle via circulating lactate, Nature, 551 (2017) 115-118.

31. A.R. Terry, N. Hay, Fuelling cancer cells, Nature reviews. Endocrinology, 15 (2019) 71-72.

32. M.R. Galdiero, G. Varricchi, G. Marone, The immune network in thyroid cancer, Oncoimmunology, 5 (2016) e1168556.

33. M. Chen, S. Hu, Y. Li, T.T. Jiang, H. Jin, L. Feng, Targeting nuclear acid-mediated immunity in cancer immune checkpoint inhibitor therapies, Signal transduction and targeted therapy, 5 (2020) 270.

34. V. Gunda, B. Gigliotti, D. Ndishabandi, T. Ashry, M. McCarthy, Z. Zhou, S. Amin, G.J. Freeman, A. Alessandrini, S. Parangi, Combinations of BRAF inhibitor and anti-PD-1/PD-L1 antibody improve survival and tumour immunity in an immunocompetent model of orthotopic murine anaplastic thyroid cancer, British journal of cancer, 119 (2018) 1223-1232.

35. J.M. Mehnert, A. Varga, M.S. Brose, R.R. Aggarwal, C.C. Lin, A. Prawira, F. de Braud, K. Tamura, T. Doi, S.A. Piha-Paul, J. Gilbert, S. Saraf, P. Thanigaimani, J.D. Cheng, B. Keam, Safety and antitumor activity of the anti-PD-1 antibody pembrolizumab in patients with advanced, PD-L1-positive papillary or follicular thyroid cancer, BMC cancer, 19 (2019) 196.

36. J. Capdevila, L.J. Wirth, T. Ernst, S. Ponce Aix, C.C. Lin, R. Ramlau, M.O. Butler, J.P. Delord, H. Gelderblom, P.A. Ascierto, A. Fasolo, D. Führer, M.L. Hütter-Krönke, P.M. Forde, A. Wrona, A. Santoro, P.M. Sadow, S. Szpakowski, H. Wu, G. Bostel, J. Faris, S. Cameron, A. Varga, M. Taylor, PD-1 Blockade in Anaplastic Thyroid Carcinoma, Journal of clinical oncology : official journal of the American Society of Clinical Oncology, 38 (2020) 2620-2627. 
Figures

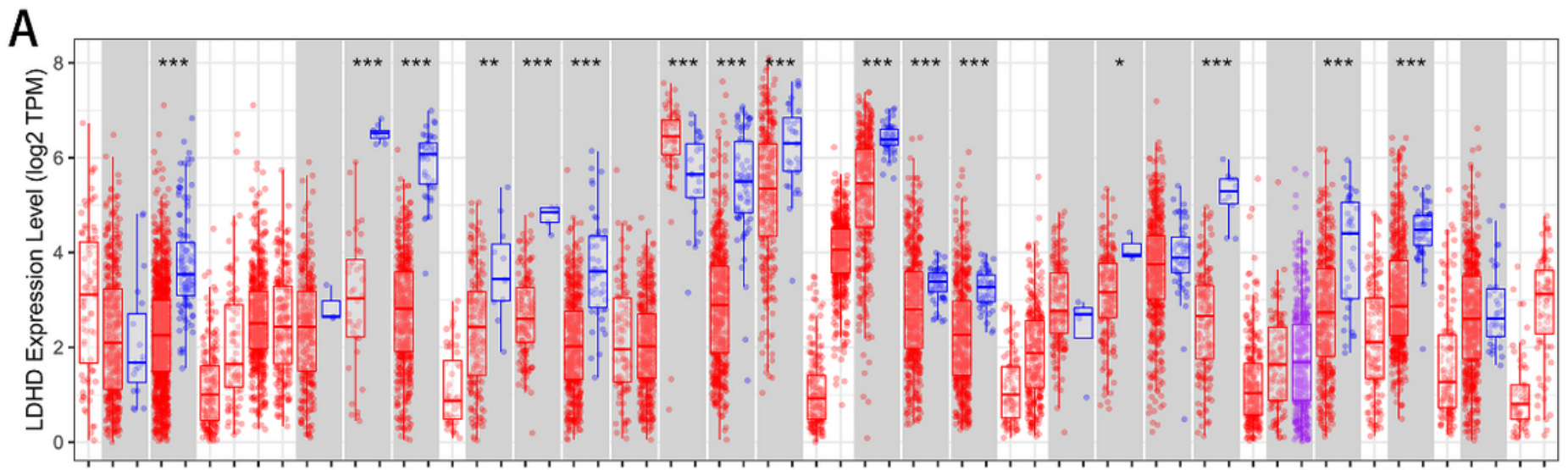

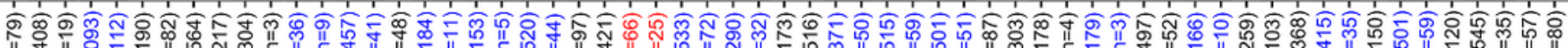

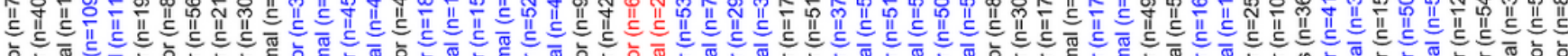

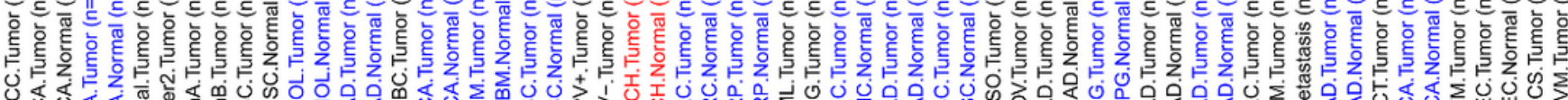

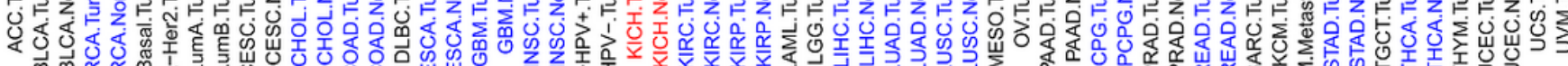

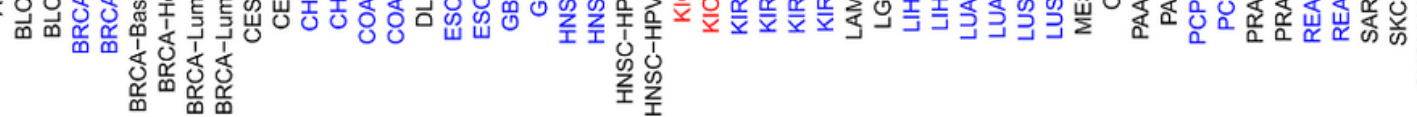
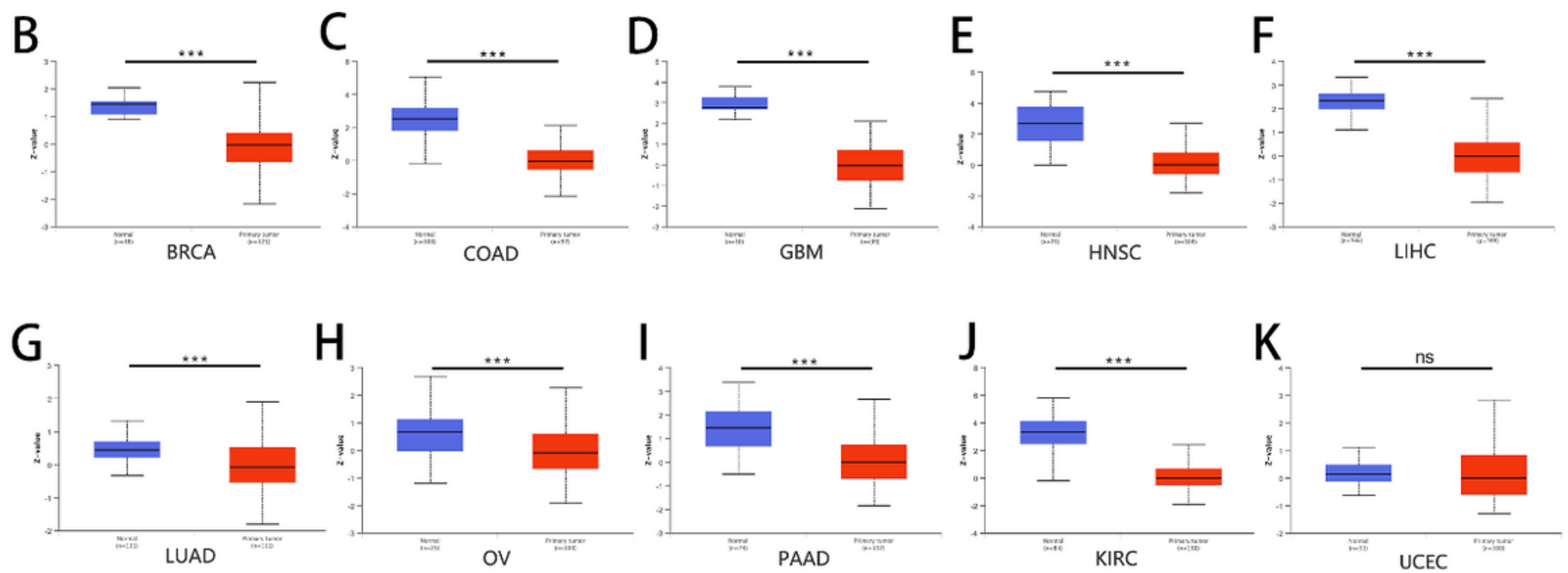

Figure 1

mRNA and protein expression of LDHD in pan-cancer. (A) The LDHD expression was significantly decreased in fifteen cancer types and increased in one cancer type compared to normal tissues. (B-K) The LDHD protein expression level was significantly decreased in nine cancer types compared to normal tissues. The red and blue boxes indicate cancer and normal tissues, individually. $* P<0.05, * * P<0.01$, and $* * * P<0.001$.

Figure 2 
Kaplan-Meier survival curves compare the strong and weak expression of LDHD in diverse cancer types. LDHD expression was significantly correlated with prognosis in the PrognoScan (A-E) and Kaplan-Meier plotter databases (F-O).
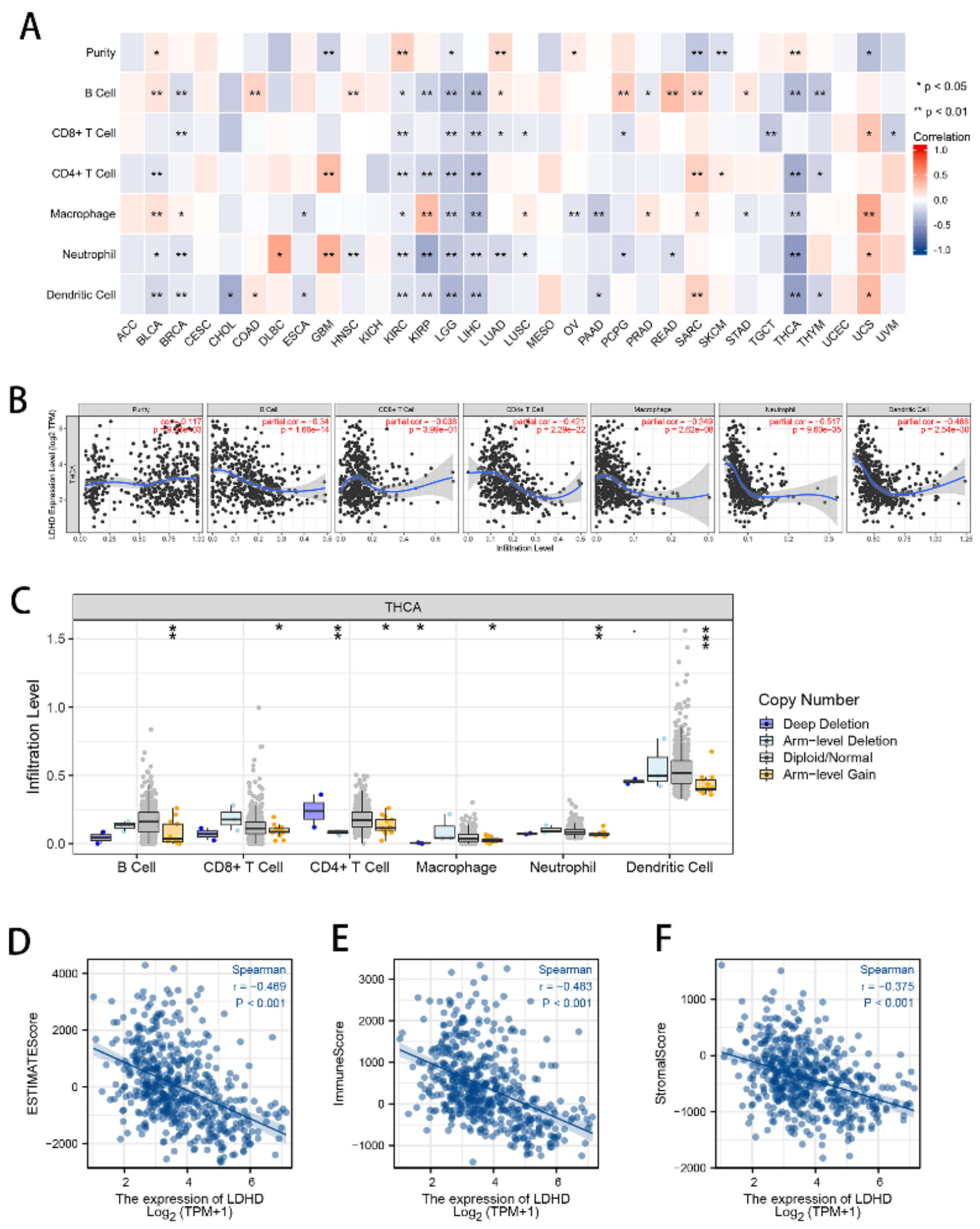

Figure 3 
Relationship of LDHD expression with immune infiltration level in THCA. (A) The correlation of LDHD expression and immune infiltration in pan-cancer. (B-C) The association of LDHD expression and immune cell infiltration in THCA. (D-F) The relationship between LDHD expression levels and the ImmuneScore, ESTIMATEScore, and StromalScore in THCA. $* P<0.05, * * P<0.01$, and $* * * P<0.001$.
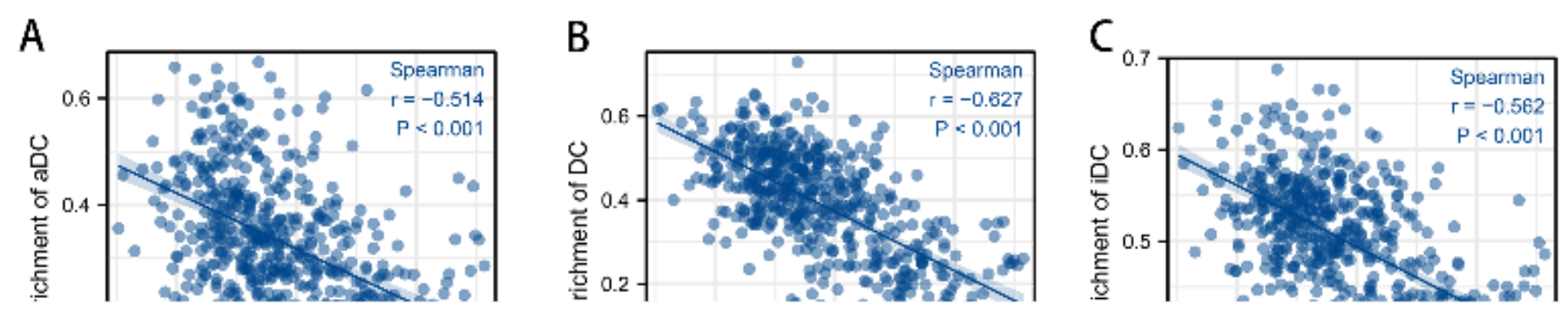

Figure 4 
Relationship of LDHD expression level and 24 immune cells type infiltration level in THCA. (A-H) LDHD expression is significantly negative relevant to infiltrating of aDC cells, DC cells, iDC cells, macrophages, neutrophils, Th1 cells, Th2 cells, and TReg in THCA. (I) Correlation of LDHD expression and 24 immune cells type infiltration in THCA.

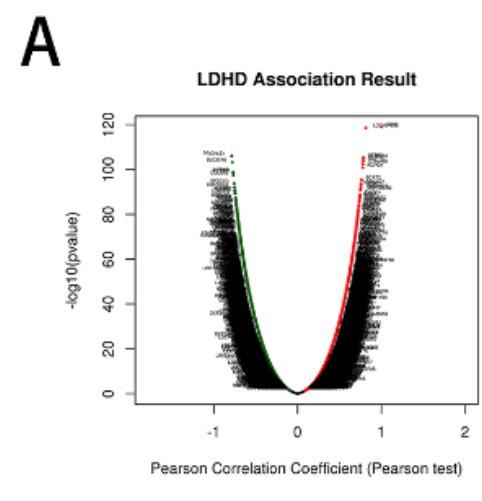

D

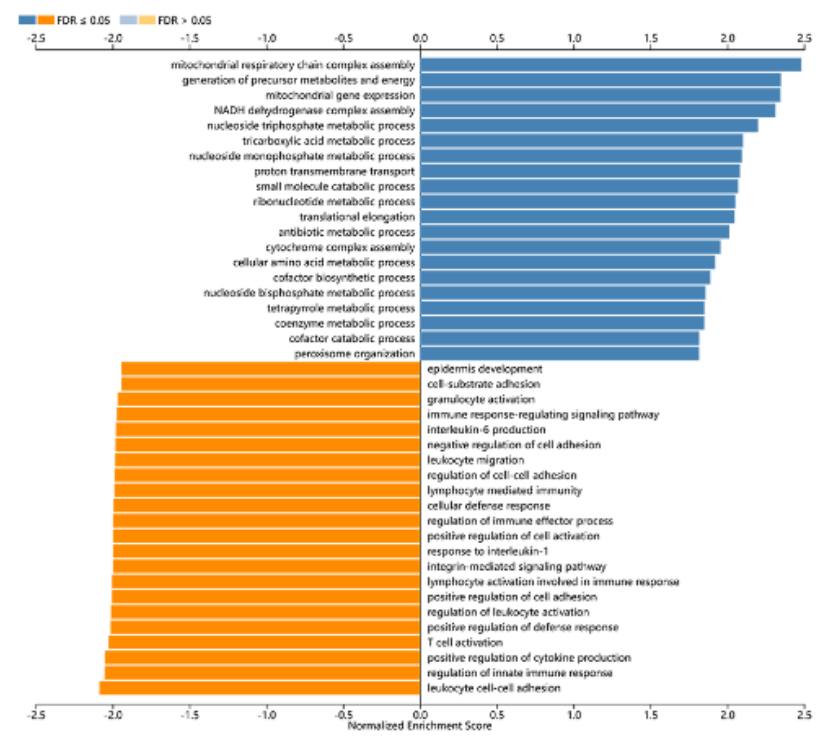

B

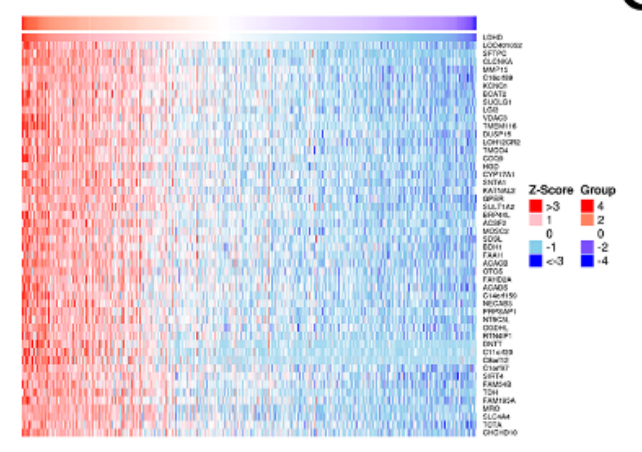

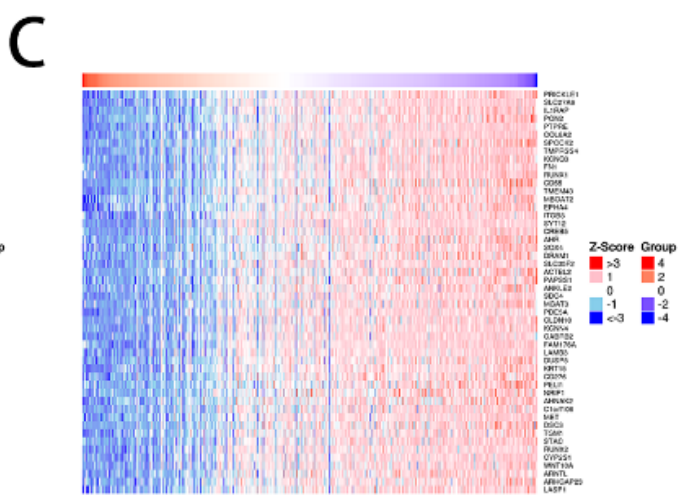

$\mathrm{E}$

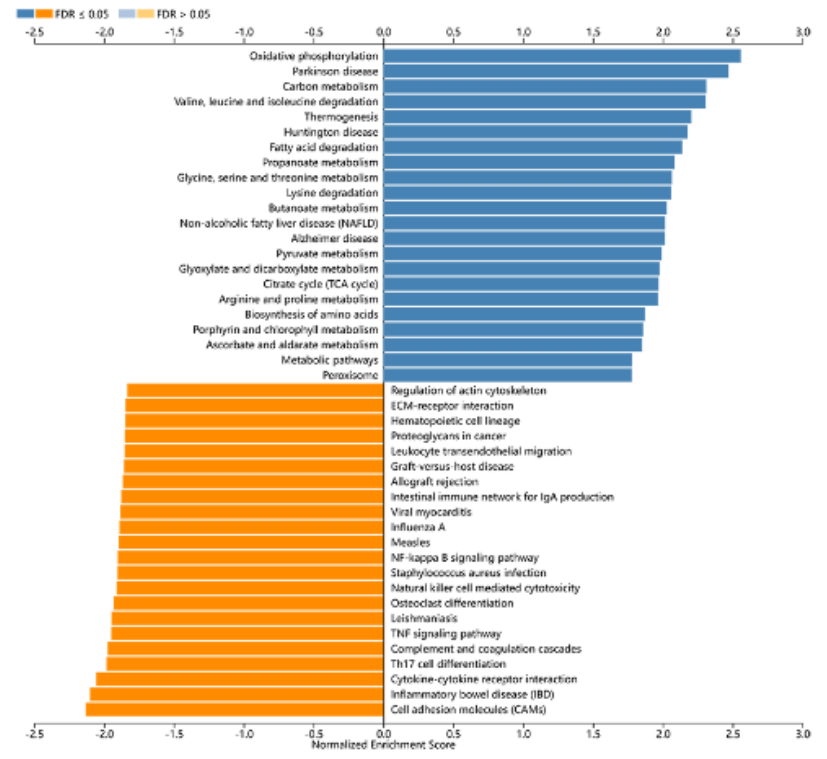

Figure 5

Co-expressed genes and functional enrichment analysis of LDHD (LinkedOmics). (A) Association of LDHD and differentially expressed genes in THCA. (B-C) The Heatmap illustrates the first 50 genes in THCA that are positively and negatively related to LDHD, respectively. GO_BP (D) and KEGG pathway (E) analyses of LDHD. Results correlation was tested by the Pearson correlation coefficient. 

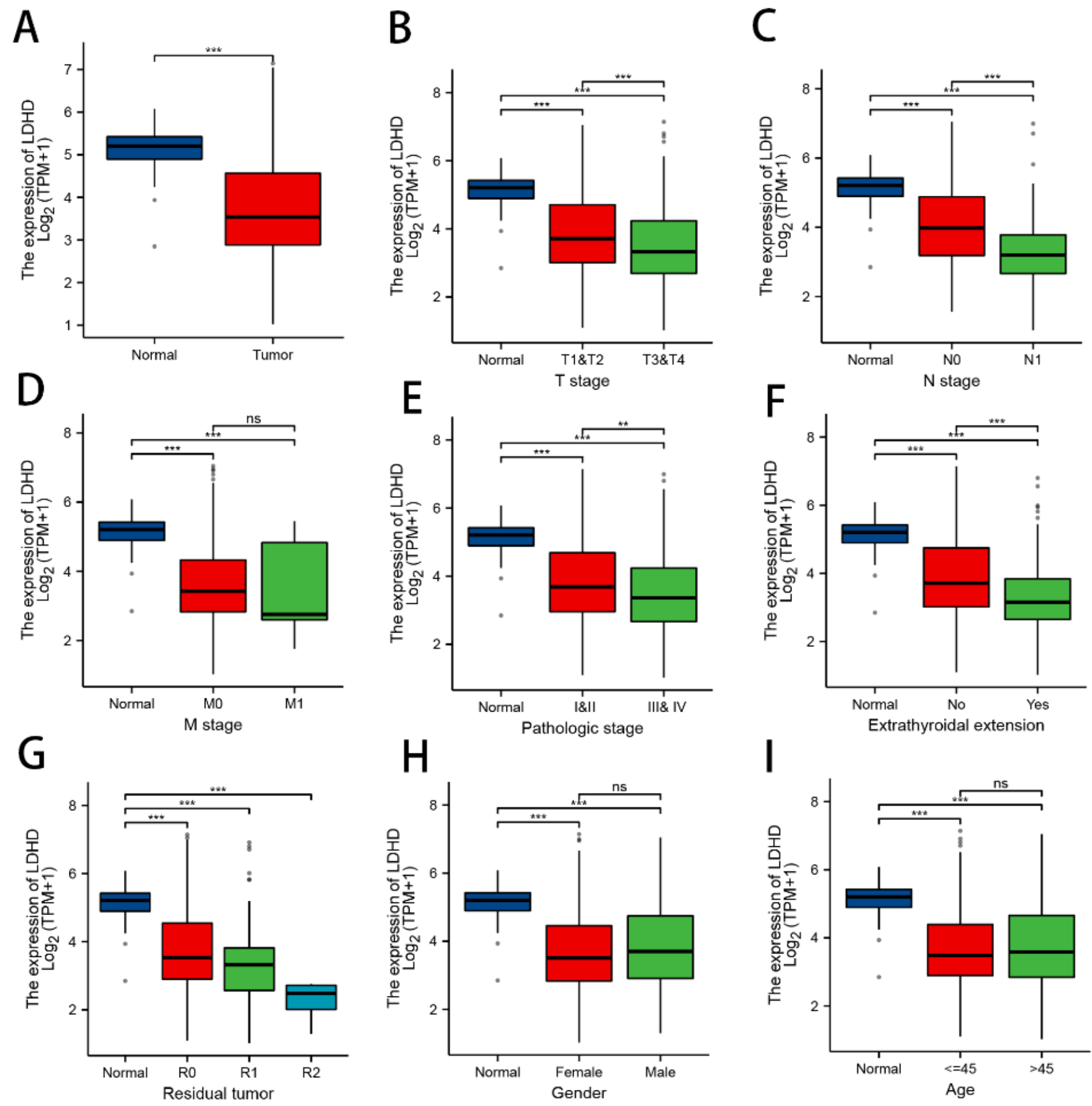

Figure 6

Correlation between LDHD expressions with clinicopathological characteristics of THCA. LDHD expression is strongly related with T stage (B), lymph node metastatic status(C), TNM stage (E), and extrathyroidal extension $(\mathrm{F}) . * P<0.05, * * P<0.01$, and $* * * P<0.001$. 

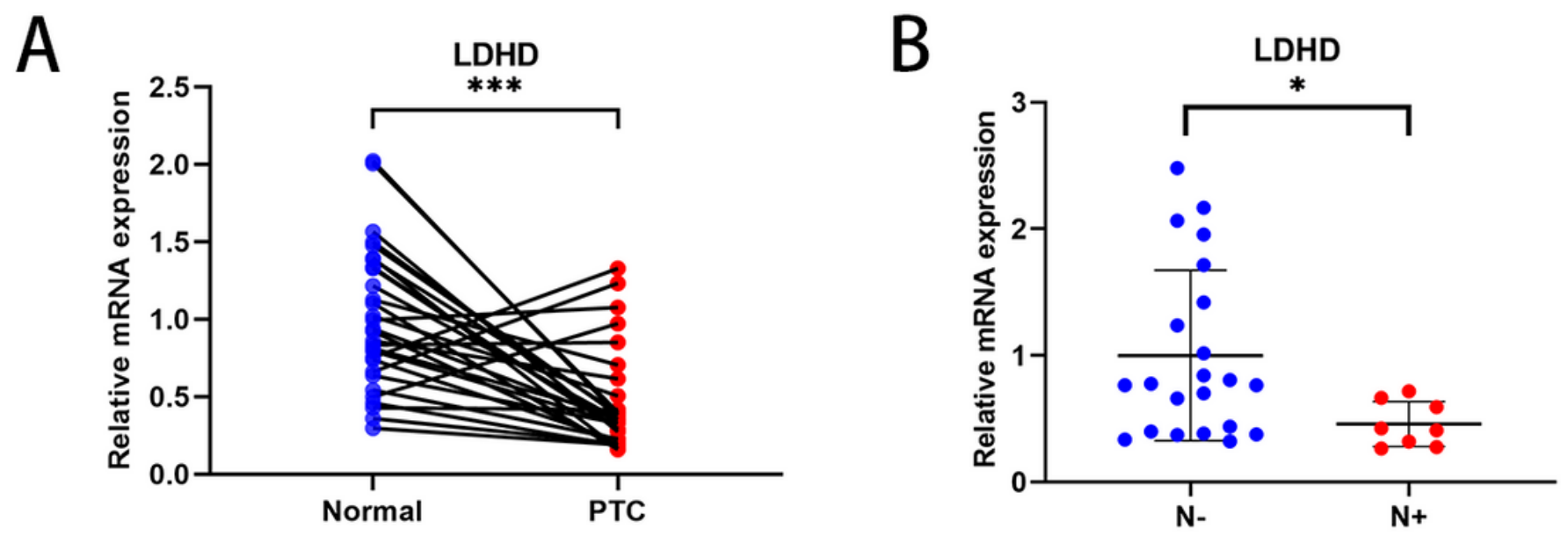

$\begin{array}{lllllllllll}\text { C } & \text { A1 } & \text { T1 } & \text { A2 } & \text { T2 } & \text { A3 } & \text { T3 } & \text { A4 } & \text { T4 } & \text { A5 } & \text { T5 }\end{array}$

LDHD
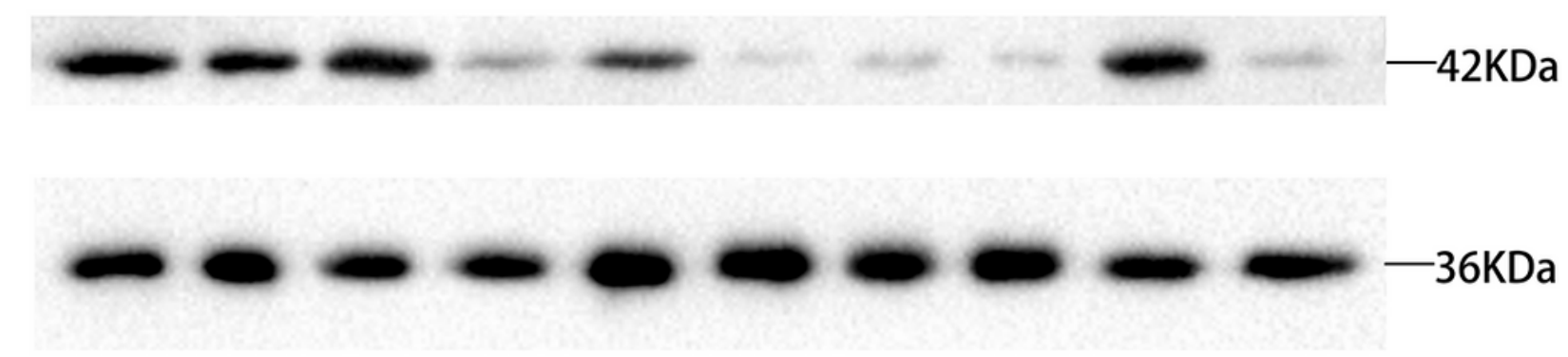

$-36 \mathrm{KDa}$

D

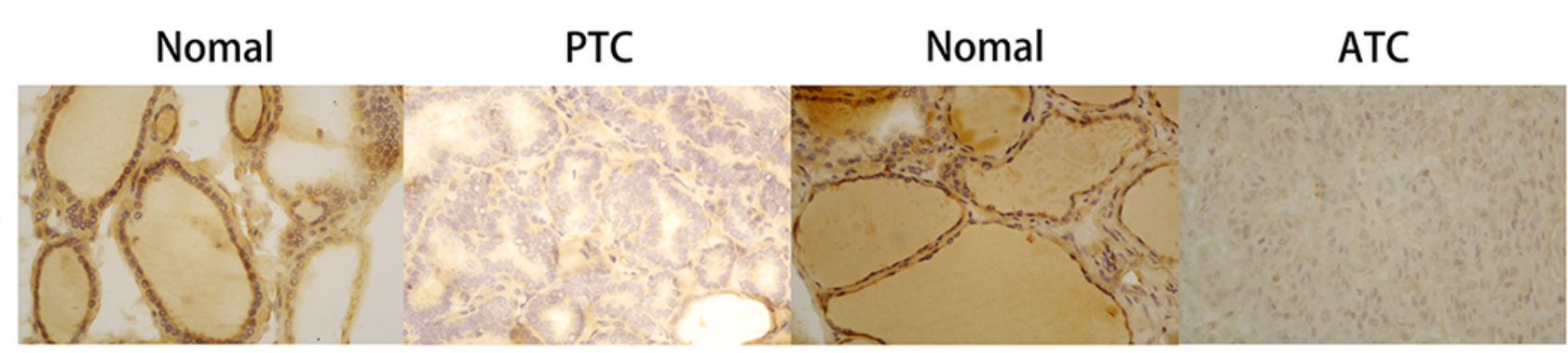

Figure 7

LDHD expression in THCA and para-carcinoma tissues. (A) The relative mRNA expression level of LDHD was significantly lower in tumor tissues compared with normal tissues based on the qRT-PCR result $(n=30)$. (B) The relative mRNA expression level of LDHD was significantly higher in negative lymph node $(n=8)$ THCA tissues compare with positive lymph node $(n=22)$ THCA tissues based on the qRT-PCR result. (C) The relative protein expression level of LDHD was significantly lower in tumor tissues compared with normal tissues based on the WB result. (D) IHC staining showed low LDHD expression in PTC and ATC tissues compared with para-carcinoma tissues. $* P<0.05, * * P<0.01$, and $* * * P<0.001$.

\section{Supplementary Files}


This is a list of supplementary files associated with this preprint. Click to download.

- FIG7C.tif

- FIGS2.jpg

- FIG7D3.tif

- FIG7D4.tif

- PCR.xIsx

- Supplementarymaterial.docx

- FIGS1.jpg 